KYUNGPOOK Math. J. 49(2009), 167-181

\title{
Geometric Means of Positive Operators
}

\section{NoBORU NAKAMURA}

Fujikoshi-kogyo Senior Highschool, Higashi-ishigane 7-5, Toyama, 930-0964, Japan e-mail : nakamura@fujikoshiko.ac.jp

Abstract. Based on Ricatti equation $X A^{-1} X=B$ for two (positive invertible) operators $A$ and $B$ which has the geometric mean $A \sharp B$ as its solution, we consider a cubic equation

$$
X(A \sharp B)^{-1} X(A \sharp B)^{-1} X=C
$$

for $A, B$ and $C$. The solution $X=(A \sharp B) \sharp_{\frac{1}{3}} C$ is a candidate of the geometric mean of the three operators. However, this solution is not invariant under permutation unlike the geometric mean of two operators. To supply the lack of the property, we adopt a limiting process due to Ando-Li-Mathias. We define reasonable geometric means of $k$ operators for all integers $k \geq 2$ by induction. For three positive operators, in particular, we define the weighted geometric mean as an extension of that of two operators.

\section{Introduction}

The quadratic equation

$$
X A^{-1} X=B
$$

for (positive invertible) operator $A$ and $B$ on a Hilbert space $H$ is said the Ricatti equation [17], which has a unique solution

$$
A \sharp B=A^{\frac{1}{2}}\left(A^{-\frac{1}{2}} B A^{-\frac{1}{2}}\right)^{\frac{1}{2}} A^{\frac{1}{2}}
$$

and this operator is defined as the geometric mean of $A$ and $B$ [2]. As its extension, the weighted geometric (or $\alpha$-power) mean $A \sharp_{\alpha} B$ for $0 \leq \alpha \leq 1$ is defined [15] by

$$
A \sharp_{\alpha} B=A^{\frac{1}{2}}\left(A^{-\frac{1}{2}} B A^{-\frac{1}{2}}\right)^{\alpha} A^{\frac{1}{2}} \text {. }
$$

The geometric means for more than two operators have been already defined by many authors [1], [3], [8], [14], etc. We here want to introduce a new geometric mean, extending the notion of the Ricatti equation. Consider the equation

$$
X(A \sharp B)^{-1} X(A \sharp B)^{-1} X=C
$$

Received 30 January 2008; revised 20 May 2008; accepted 16 May 2008.

2000 Mathematics Subject Classification: 47A63, 47A64.

Key words and phrases: positive operator, geometric mean, arithmetic-geometric mean inequality, reverse inequality.

Dedicated to the memory of Emeritus Professor Masahiro Nakamura. 
for operator $A, B$ and $C$. Then we easily have a unique solution which is given by

$$
X=(A \sharp B) \sharp_{\frac{1}{3}} C .
$$

If $A, B, C$ commute with each other, then the above operator $X$ is reduced to $(A B C)^{1 / 3}$, so that it seems a candidate of the geometric mean. But the operator lacks the property $\mathrm{P} 3$ below, i.e., permutation invariance for $k=3$ to be a reasonable geometric mean. The following properties were postulated for a geomtric mean $G\left(A_{1}, A_{2}, \cdots, A_{k}\right)=G_{k}\left(A_{1}, A_{2}, \cdots, A_{k}\right)$ of $k$ operators in [3]:

P1 Consistency with scalars. If $A_{1}, A_{2}, \cdots, A_{k}$ commute then

$$
G\left(A_{1}, A_{2}, \cdots, A_{k}\right)=\left(A_{1} A_{2} \cdots A_{k}\right)^{\frac{1}{k}} .
$$

P1' This implies $G(\overbrace{A, \cdots, A}^{k})=A$.

P2 Joint homogeneity.

$G\left(a_{1} A_{1}, a_{2} A_{2}, \cdots, a_{k} A_{k}\right)=\left(a_{1} a_{2} \cdots a_{k}\right)^{\frac{1}{k}} G\left(A_{1}, A_{2}, \cdots, A_{k}\right) \quad$ for $a_{i} \geq 0$ with $i=1, \cdots, k$.

P2' This implies $G\left(a A_{1}, a A_{2}, \cdots, a A_{k}\right)=a G\left(A_{1}, A_{2}, \cdots, A_{k}\right) \quad(a \geq 0)$.

P3 Permutation invariance. For any permutation $\pi\left(A_{1}, A_{2}, \cdots, A_{k}\right)$ of $\left(A_{1}, A_{2}, \cdots, A_{k}\right), G\left(A_{1}, A_{2}, \cdots, A_{k}\right)=G\left(\pi\left(A_{1}, A_{2}, \cdots, A_{k}\right)\right)$.

P4 Monotonicity. The map $\left(A_{1}, A_{2}, \cdots, A_{n}\right) \mapsto G\left(A_{1}, A_{2}, \cdots, A_{n}\right)$ is monotone, i.e., if $A_{i} \geq B_{i}$ for $i=1, \cdots, k$, then $G\left(A_{1}, A_{2}, \cdots, A_{k}\right) \geq G\left(B_{1}, B_{2}, \cdots, B_{k}\right)$.

P5 Continuity from above. If $\left\{A_{1}^{(n)}\right\},\left\{A_{2}^{(n)}\right\}, \cdots,\left\{A_{k}^{(n)}\right\}$ are monotonic decreasing sequences converging to $A_{1}, A_{2}, \cdots, A_{k}$, respectively, then $\left\{G\left(A_{1}^{(n)}, A_{2}^{(n)}, \cdots, A_{k}^{(n)}\right)\right\}$ converges to $G\left(A_{1}, A_{2}, \cdots, A_{k}\right)$.

P6 Congruence invariance. For any invertible $S$,

$$
G\left(S^{*} A_{1} S, S^{*} A_{2} S, \cdots, S^{*} A_{k} S\right)=S^{*} G\left(A_{1}, A_{2}, \cdots, A_{k}\right) S .
$$

P7 Joint concavity. The map $\left(A_{1}, A_{2}, \cdots, A_{k}\right) \mapsto G\left(A_{1}, A_{2}, \cdots, A_{k}\right)$ is jointly concave:

$$
\begin{aligned}
& G\left(\lambda A_{1}+(1-\lambda) A_{1}^{\prime}, \lambda A_{2}+(1-\lambda) A_{2}^{\prime}, \cdots, \lambda A_{k}+(1-\lambda) A_{k}^{\prime}\right) \\
\geq & \lambda G\left(A_{1}, A_{2}, \cdots, A_{k}\right)+(1-\lambda) G\left(A_{1}^{\prime}, A_{2}^{\prime}, \cdots, A_{k}^{\prime}\right) \quad(0<\lambda<1) .
\end{aligned}
$$


P8 Self-duality. $G\left(A_{1}, A_{2}, \cdots, A_{k}\right)^{*}=G\left(A_{1}, A_{2}, \cdots, A_{k}\right)$. The dual $G\left(A_{1}, A_{2}, \cdots, A_{k}\right)^{*}$ is defined by

$$
G\left(A_{1}, A_{2}, \cdots, A_{k}\right)^{*}=G\left(A_{1}^{-1}, A_{2}^{-1}, \cdots, A_{k}^{-1}\right)^{-1} .
$$

P9 (In case $A_{1}, A_{2}, \cdots, A_{k}$ are matrices.) Determinant identity.

$$
\operatorname{det} G\left(A_{1}, A_{2}, \cdots, A_{k}\right)=\left(\operatorname{det} A_{1} \cdot \operatorname{det} A_{2} \cdots \cdot \operatorname{det} A_{k}\right)^{\frac{1}{k}} .
$$

P10 The arithmetic-geometric-harmonic mean inequaility.

$$
\frac{A_{1}+A_{2}+\cdots+A_{k}}{k} \geq G\left(A_{1}, A_{2}, \cdots, A_{k}\right) \geq\left(\frac{A_{1}^{-1}+A_{2}^{-1}+\cdots+A_{k}^{-1}}{k}\right)^{-1} .
$$

Mentioned as before, we can show that the operator defined by (1.2) does not satisfy the basic property P3. Supplying this fact, we employ the iteration technique due to Ando-Li-Mathias [3]: We define the three sequences $\left\{A_{n}\right\},\left\{B_{n}\right\}$ and $\left\{C_{n}\right\}$, for example, by $A_{1}=A, B_{1}=B, C_{1}=C$,

$$
\begin{aligned}
& A_{n+1}=\left(B_{n} \sharp C_{n}\right) \sharp_{\frac{1}{3}} A_{n}=A_{n} \sharp_{\frac{2}{3}}\left(B_{n} \sharp C_{n}\right) \quad \text { (see WG3), } \\
& B_{n+1}=B_{n} \sharp_{\frac{2}{3}}\left(C_{n} \sharp A_{n}\right) \quad \text { and } \\
& C_{n+1}=C_{n} \sharp_{\frac{2}{3}}\left(A_{n} \sharp B_{n}\right) \quad \text { for } n \geq 1 .
\end{aligned}
$$

Then we see that $\left\{A_{n}\right\},\left\{B_{n}\right\}$ and $\left\{C_{n}\right\}$ converge and have a common limit. Now we may define the limit as the desired geometric mean of the operators $A, B$ and $C$, which posseses all properties $\mathrm{P} 1-\mathrm{P} 10$. In this paper we define a geometric mean, somewhat different from that presented in [3] of $k(\geq 3)$ operators which satisfies the above properties P1-P10. For three operators we define a weighted geometric mean of operators, which really extends that of two operators.

All operators are assumed positive invertible if stated otherwise.

\section{Definition of geometric mean of more than two operators}

Before we define a geometric mean of $k$ operators, we want to state some useful facts for our discussion. First we introduce the metric $d(A, B)$, called Thompson metric on the positive cone $\Omega$ of all (positive invertible) operators defined ([18], [4], [5], [7]) by

$$
d(A, B)=\max \{\log M(A / B), \log M(B / A)\} \quad \text { for } A, B \in \Omega,
$$

where

$$
M(A / B)=\inf \{\lambda>0: A \leq \lambda B\}=\left\|B^{-1 / 2} A B^{-1 / 2}\right\| .
$$

We remark that $\Omega$ is complete with respect to the corresponding metric topology [7]. As a basic inequality between weighted geometric means of two operators, the 
following holds [4]:

WG0 $\quad d\left(A_{1} \sharp_{\alpha} A_{2}, B_{1} \sharp_{\alpha} B_{2}\right) \leq(1-\alpha) d\left(A_{1}, B_{1}\right)+\alpha d\left(A_{2}, B_{2}\right)$

for $A_{1}, A_{2}, B_{1}, B_{2} \in \Omega$ and $\alpha \in(0,1)$. Next for convenience sake, we, parallel to P1-P10, state basic properties of the weighted geometric mean $A \sharp_{\alpha} B$ defined by (1.1) $[2],[11]$ :

WG1 $\quad A \sharp_{\alpha} A=A$.

WG2 $\quad(a A) \sharp_{\alpha}(b B)=a^{1-\alpha} b^{\alpha} A \sharp_{\alpha} B$.

WG3 $\quad A \sharp_{\alpha} B=B \sharp_{1-\alpha} A$.

WG4 $A \sharp_{\alpha} B$ is monotone, i.e., if $A \geq C$ and $B \geq D$, then $A \sharp_{\alpha} B \geq C \sharp_{\alpha} D$.

WG5 The map $(A, B) \mapsto A \sharp_{\alpha} B$ is continuous from above.

WG6 $A \sharp_{\alpha} B$ is invariant with respect to congruence, i.e., $S^{*}\left(A \sharp_{\alpha} B\right) S=S^{*} A S \sharp_{\alpha} S^{*} B S$ for any invertible operator $S$.

WG7 The map $(A, B) \mapsto A \sharp_{\alpha} B$ is jointly concave.

WG8 $A \sharp_{\alpha} B$ is self-dual, i.e., $A \sharp_{\alpha} B=\left(A^{-1} \sharp_{\alpha} B^{-1}\right)^{-1}$.

WG9 (In case $A$ and $B$ are matrices, $) \operatorname{det}\left(A \sharp_{\alpha} B\right)=(\operatorname{det} A)^{1-\alpha}(\operatorname{det} B)^{\alpha}$.

WG10 The weighted arithmetic-geometric-harmonic mean inequality holds:

$$
(1-\alpha) A+\alpha B \geq A \sharp_{\alpha} B \geq\left((1-\alpha) A^{-1}+\alpha B^{-1}\right)^{-1} .
$$

The following fact $[12,(11)]$ is also useful, so we add it to the above properties: WG11

$$
A \sharp_{\alpha}\left(A \sharp_{\beta} B\right)=A \sharp_{\alpha \beta} B \text {. }
$$

Now we want to define our geometric means for all integers $k \geq 2$.

Definition 2.1. (1) First for $k=2$, define $G\left(A_{1}, A_{2}\right)=A_{1} \sharp A_{2}$ (the usual geometric mean) for two operators $A_{1}$ and $A_{2}$. Then $G\left(A_{1}, A_{2}\right)$ satisfies all properties P1-P10, and moreover, from WG0, between two geometric means $G\left(A_{1}, A_{2}\right)$ and $G\left(B_{1}, B_{2}\right)$ the following inequality holds:

$$
d\left(G\left(A_{1}, A_{2}\right), G\left(B_{1}, B_{2}\right)\right) \leq \frac{1}{2}\left(d\left(A_{1}, B_{1}\right)+d\left(A_{2}, B_{2}\right)\right) .
$$

To define geometric means for $k \geq 3$ by induction, we assume that for $k$ operators $A_{1}, \cdots, A_{k}$ we have obtained a geometric mean $G\left(A_{1}, \cdots, A_{k}\right)$ such that the mean 
satisfies all properties P1-P10, and the following inequality, for another $k$-tuple of operators $B_{1}, \cdots, B_{k}$, holds:

$$
d\left(G\left(A_{1}, \cdots, A_{k}\right), G\left(B_{1}, \cdots, B_{k}\right)\right) \leq \frac{1}{k} \sum_{i=1}^{k} d\left(A_{i}, B_{i}\right) .
$$

(2) Then we shall define a geometric mean $G\left(A_{1}, \cdots, A_{k+1}\right)$ of $(k+1)$ operators $A_{1}, \cdots, A_{k+1}$ as the common limit of the sequences $\left\{A_{i}^{(r)}\right\}_{r=1}^{\infty}(i=1, \cdots, k+1)$ defined by

$$
A_{i}^{(1)}=A_{i} \text { and } A_{i}^{(r+1)}=A_{i}^{(r)} \sharp_{\frac{k-1}{k}} G\left(\left(A_{j}^{(r)}\right)_{j \neq i}\right) \text { for } r \geq 1 \text {. }
$$

Here

$$
G\left(\left(A_{j}^{(r)}\right)_{j \neq i}\right)=G\left(A_{1}^{(r)}, \cdots, A_{i-1}^{(r)}, A_{i+1}^{(r)}, \cdots, A_{k+1}^{(r)}\right) .
$$

Now we have to show:

Theorem 2.2. For a fixed $k \geq 2$, assume $G\left(A_{1}, \cdots, A_{k}\right)$ is defined and satisfies P1-P10 and (2.2). Then the $(k+1)$ sequences $\left\{A_{i}^{(r)}\right\}_{r=1}^{\infty}(i=1, \cdots, k+1)$ defined by (2.3) are convergent in the Thompson metric and have a common limit. The limit defined as the geometric mean $G\left(A_{1}, \cdots, A_{k+1}\right)$ satisfies all properties P1-P10 and the inequality $(2.2)$ for $(k+1)$ operators $A_{1}, \cdots, A_{k+1}$.

Proof. First for $d\left(A_{i}^{(r+1)}, A_{i}^{(r)}\right)(i=1, \cdots, k+1)$, we have

$$
d\left(A_{i}^{(r+1)}, A_{i}^{(r)}\right) \leq \frac{1}{k+1} \sum_{j=1, j \neq i}^{k+1} d\left(A_{i}^{(r)}, A_{j}^{(r)}\right) .
$$

In fact, from P1', P3 and (2.2)

$$
\begin{aligned}
& d\left(A_{i}^{(r+1)}, A_{i}^{(r)}\right)=d\left(A_{i}^{(r)_{\sharp k}} G\left(\left(A_{j}^{(r)}\right)_{j \neq i}\right), A_{i}^{(r)}\right) \\
& =d\left(A_{i}^{(r)} \sharp_{\frac{k}{k+1}} G\left(A_{1}^{(r)}, \cdots, A_{i-1}^{(r)}, A_{i+1}^{(r)}, \cdots, A_{k+1}^{(r)}\right), A_{i}^{(r)} \sharp_{k} G\left(A_{i}^{(r)}, \cdots, A_{i}^{(r)}\right)\right) \\
& \leq \frac{1}{k+1} d\left(A_{i}^{(r)}, A_{i}^{(r)}\right)+\frac{k}{k+1} \cdot \frac{1}{k}\left(d\left(A_{1}^{(r)}, A_{i}^{(r)}\right)+d\left(A_{2}^{(r)}, A_{i}^{(r)}\right)\right. \\
& \left.\quad+\cdots+d\left(A_{i-1}^{(r)}, A_{i}^{(r)}\right)+d\left(A_{i+1}^{(r)}, A_{i}^{(r)}\right)+\cdots+d\left(A_{k+1}^{(r)}, A_{i}^{(r)}\right)\right) \\
& =\frac{1}{k+1} \sum_{j=1, j \neq i}^{k+1} d\left(A_{j}^{(r)}, A_{i}^{(r)}\right)=\frac{1}{k+1} \sum_{j=1, j \neq i}^{k+1} d\left(A_{i}^{(r)}, A_{j}^{(r)}\right) .
\end{aligned}
$$


For $d\left(A_{i}^{(r+1)}, A_{j}^{(r+1)}\right)(i \neq j)$, say, for $i<j$, we have

$$
\begin{aligned}
& d\left(A_{i}^{(r+1)}, A_{j}^{(r+1)}\right)=d\left(A_{i}^{(r)} \sharp_{k} G\left(\left(A_{l}^{(r)}\right)_{l \neq i}\right), A_{j}^{(r)_{\frac{k}{k}}} G\left(\left(A_{l}^{(r)}\right)_{l \neq j}\right)\right) \\
& =d\left(A_{i}^{(r)} \sharp_{k} G\left(A_{j}^{(r)}, A_{1}^{(r)}, \cdots, A_{i-1}^{(r)}, A_{i+1}^{(r)}, \cdots, A_{j-1}^{(r)}, A_{j+1}^{(r)}, \cdots, A_{k+1}^{(r)}\right),\right. \\
& \left.\quad A_{j}^{(r)} \sharp_{\frac{k}{k+1}} G\left(A_{i}^{(r)}, A_{1}^{(r)}, \cdots, A_{i-1}^{(r)}, A_{i+1}^{(r)}, \cdots, A_{j-1}^{(r)}, A_{j+1}^{(r)}, \cdots, A_{k+1}^{(r)}\right)\right) \\
& \leq \frac{1}{k+1} d\left(A_{i}^{(r)}, A_{j}^{(r)}\right)+\frac{k}{k+1} \cdot \frac{1}{k} d\left(A_{j}^{(r)}, A_{i}^{(r)}\right)=\frac{2}{k+1} d\left(A_{i}^{(r)}, A_{j}^{(r)}\right) .
\end{aligned}
$$

Hence we have

$$
\begin{aligned}
& d\left(A_{i}^{(r+1)}, A_{j}^{(r+1)}\right) \leq \frac{2}{k+1} d\left(A_{i}^{(r)}, A_{j}^{(r)}\right) \\
& \leq\left(\frac{2}{k+1}\right)^{2} d\left(A_{i}^{(r-1)}, A_{j}^{(r-1)}\right) \leq \cdots \leq\left(\frac{2}{k+1}\right)^{r} d\left(A_{i}, A_{j}\right) .
\end{aligned}
$$

From (2.4) and the above inequality, we then have

$$
d\left(A_{i}^{(r+1)}, A_{i}^{(r)}\right) \leq \frac{1}{k+1} \sum_{j=1, j \neq i}^{k+1}\left(\frac{2}{k+1}\right)^{r-1} d\left(A_{i}, A_{j}\right)=\left(\frac{2}{k+1}\right)^{r-1} K_{i},
$$

where $K_{i}=\frac{1}{k+1} \sum_{j=1, j \neq i}^{k+1} d\left(A_{i}, A_{j}\right)$. Hence for any $r, s$ such that $r \geq s$

$$
\begin{aligned}
& d\left(A_{i}^{(r+1)}, A_{i}^{(s)}\right) \leq \sum_{l=s}^{r} d\left(A_{i}^{(l+1)}, A_{i}^{(l)}\right) \\
& \leq \sum_{l=s}^{r}\left(\frac{2}{k+1}\right)^{l-1} K_{i} \leq \frac{(k+1) K_{i}}{k-1}\left(\frac{2}{k+1}\right)^{s-1} \rightarrow 0(\text { as } s \rightarrow \infty) .
\end{aligned}
$$

This implies that $\left\{A_{i}^{(r)}\right\}$ (for all $i=1, \ldots, k+1$ ) are convergent, and then from (2.5) their limits are identical. For the properties P1-P10 of $G\left(A_{1}, \cdots, A_{k+1}\right)$, it is not difficult to show them. For example, to see P3, let

$$
\left(B_{1}, \cdots, B_{k+1}\right)=\pi\left(A_{1}, \cdots, A_{k+1}\right)\left(=\left(A_{\pi(1)}, \cdots, A_{\pi(k+1)}\right)\right)
$$

be a permutation of $\left(A_{1}, \cdots, A_{k+1}\right)$. Put for all $i=1, \cdots, k+1$

$$
B_{i}^{(1)}=B_{i}=A_{\pi(i)} \text { and } B_{i}^{(r+1)}=B_{i}^{(r)_{\sharp k}} G\left(\left(B_{j}^{(r)}\right)_{j \neq i}\right) \text { for } r \geq 1 .
$$

Then we can see that

$$
B_{i}^{(r)}=A_{\pi(i)}^{(r)} \text { for } r \geq 1, \quad\left(A_{\pi(i)}^{(r)} \text { is defined before by }(2.3)\right),
$$


so that $\left(B_{1}^{(r)}, \cdots, B_{k+1}^{(r)}\right)$ is a rearrangement of $\left(A_{1}, \cdots, A_{k+1}\right)$. Hence all sequences $\left\{B_{i}^{(r)}\right\}_{r=1}^{\infty}$ are convergent and have the common limit $G\left(A_{1}, \cdots, A_{k+1}\right)$. This implies the desired $G\left(\pi\left(A_{1}, \cdots, A_{k+1}\right)\right)=G\left(A_{1}, \cdots, A_{k+1}\right)$.

For the inequality $(2.2)$ for two tuples $\left(A_{1}, \cdots, A_{k+1}\right)$ and $\left(B_{1}, \cdots, B_{k+1}\right)$ of operators, let $\left\{A_{i}^{(r)}\right\}(i=1, \cdots, k+1)$ be the sequences defined by $(2.3)$ and $\left\{B_{i}^{(r)}\right\}(i=1, \cdots, k+1)$ be those defined by $B_{i}^{(1)}=B_{1}$ and

$$
B_{i}^{(r+1)}=B_{i}^{(r)_{\frac{k}{k}}} G\left(\left(B_{j}^{(r)}\right)_{j \neq i}\right) \text { for } r \geq 1 .
$$

Then by the assumption (2.2), we have, for all $i=1, \cdots, k+1$

$$
\begin{aligned}
& d\left(A_{i}^{(r+1)}, B_{i}^{(r+1)}\right)=d\left(A_{i}^{(r)} \sharp_{\frac{k}{k+1}} G\left(\left(A_{j}^{(r)}\right)_{j \neq i}\right), B_{i}^{(r)} \sharp_{\frac{k}{k+1}} G\left(\left(B_{j}^{(r)}\right)_{j \neq i}\right)\right) \\
& \leq \frac{1}{k+1} d\left(A_{i}^{(r)}, B_{i}^{(r)}\right)+\frac{1}{k+1} \sum_{j=1, j \neq i}^{k+1} d\left(A_{j}^{(r)}, B_{j}^{(r)}\right)=\frac{1}{k+1} \sum_{j=1}^{k+1} d\left(A_{j}^{(r)}, B_{j}^{(r)}\right) .
\end{aligned}
$$

Thus we have

$$
\sum_{i=1}^{k+1} d\left(A_{i}^{(r+1)}, B_{i}^{(r+1)}\right) \leq \sum_{i=1}^{k+1} d\left(A_{i}^{(r)}, B_{i}^{(r)}\right) \leq \cdots \leq \sum_{i=1}^{k+1} d\left(A_{i}, B_{i}\right) .
$$

Taking the limit as $r \rightarrow \infty$, we obtain

$$
(k+1) d\left(G\left(A_{1}, \cdots, A_{k+1}\right), G\left(B_{1}, \cdots, B_{k+1}\right)\right) \leq \sum_{i=1}^{k+1} d\left(A_{i}, B_{i}\right),
$$

which is the desired.

\section{Other definitions of geometric means}

1. Ando-Li-Mathias [3] defined a geometric mean $G_{A}=G_{A}\left(A_{1}, \cdots, A_{k}\right)$ having all properties P1-P10 as follows: For $k=2$, put $G_{A}=A_{1} \sharp A_{2}$. Assuming $G_{A}$ for $k(\geq 2)$ operators, by induction, define $G_{A}\left(A_{1}, \cdots, A_{k+1}\right)$ as the common limit of the $(k+1)$ sequences $\left\{A_{i}^{(r)}\right\}_{r=1}^{\infty}(i=1, \cdots, k+1)$ such that $A_{i}^{(1)}=A_{i}$ and

$$
A_{i}^{(r+1)}=G_{A}\left(\left(A_{j}^{(r)}\right)_{j \neq i}\right) \text { for } r \geq 1 .
$$

2. Kosaki [14] defined a geometric mean $G_{K}=G_{K}\left(A_{1}, \cdots, A_{k}\right)$ as follows: First define

$$
\begin{aligned}
G_{K}{ }^{+} & =G_{K}^{+}\left(A_{1}, \cdots, A_{k}\right): \\
& =\frac{1}{(\Gamma(1 / k))^{k}} \int_{\Lambda_{k}}\left\{\sum_{j=1}^{k} \lambda_{j} A_{j}^{-1}\right\}^{-1}\left\{\sum_{j=1}^{k} \lambda_{j}\right\}\left\{\prod_{j=1}^{k} \lambda_{j}^{1 / k-1}\right\} d \lambda_{1} \cdots d \lambda_{k},
\end{aligned}
$$


where $\Lambda_{k}=\left\{\left(\lambda_{1}, \cdots, \lambda_{k}\right) ; \lambda_{j} \geq 0(j=1, \cdots, k), \sum_{j=1}^{k} \lambda_{j} \leq 1\right\}$. Then define $G_{K}^{-}=\left(G_{K}^{+}\right)^{*}$, the dual of $G_{K}^{+}$(see P8) and $G_{K}=G_{K}^{+} \sharp G_{K}^{-}$. The function $G_{K}^{+}$does not have the property $\mathrm{P} 8$-self-duality (nor P9), but $G_{K}$ has $\mathrm{P} 8$ by the modification [3].

3. Anderson-Morley-Trapp [1] defined a geometric mean $G_{\text {amt }}$ of $k(\geq 2)$ operators based on symmetric functions. For example, for three operators $A, B$ and $C$, define $A_{1}^{(1)}=A, A_{2}^{(1)}=B, A_{3}^{(1)}=C$, and for $r \geq 1$

$$
\begin{aligned}
& A_{1}^{(r+1)}=\left(A_{1}^{(r)}+A_{2}^{(r)}+A_{3}^{(r)}\right) / 3, \\
& A_{2}^{(r+1)}=\left\{A_{1}^{(r)}:\left(A_{2}^{(r)}+A_{3}^{(r)}\right)+A_{2}^{(r)}:\left(A_{3}^{(r)}+A_{1}^{(r)}\right)+A_{3}^{(r)}:\left(A_{1}^{(r)}+A_{2}^{(r)}\right)\right\} / 2, \\
& A_{3}^{(r+1)}=3\left(\left(A_{1}^{(r)}: A_{2}^{(r)}\right): A_{3}^{(r)}\right)=3\left(A_{1}^{(r)}: A_{2}^{(r)}: A_{3}^{(r)}\right) .
\end{aligned}
$$

Here $X: Y=\left(X^{-1}+Y^{-1}\right)^{-1}$ is the parallel sum of operators $X$ and $Y$. Then the sequences $\left\{A_{1}^{(r+1)}\right\},\left\{A_{2}^{(r+1)}\right\},\left\{A_{3}^{(r+1)}\right\}$ converge to a common limit, which is denoted by $G_{\text {amt }}^{+}=G_{\text {amt }}^{+}(A, B, C)$. The mean $G_{\text {amt }}$ is defined by $G_{\text {amt }}=G_{\text {amt }}^{+} \sharp G_{\text {amt }}^{-}$, where $G_{\text {amt }}^{-}=\left(G_{\text {amt }}^{+}\right)^{*}$, the dual of $G_{\text {amt }}^{+}$. None of $G_{\text {amt }}, G_{\text {amt }}^{+}, G_{\text {amt }}^{-}$satisfiy P2-joint homogeneity [3].

Denote by $G$ the geometric mean of our definition in Theorem 2.2. Then we compare $G_{A}, G_{K}, G_{\mathrm{amt}}$ and $G$ by the following three $2 \times 2$ matrices: Let

$$
A=\left[\begin{array}{ll}
2 & 1 \\
1 & 1
\end{array}\right], \quad B=\left[\begin{array}{ll}
1 & 2 \\
2 & 5
\end{array}\right] \text { and } C=\left[\begin{array}{ll}
1 & 0 \\
0 & 1
\end{array}\right]
$$

Then by numerical computation cited from [3] (less than $10^{-4}$ discarded),

$$
\begin{gathered}
G_{A}=\left[\begin{array}{ll}
0.9319 & 0.6636 \\
0.6636 & 1.5456
\end{array}\right], G_{K}=\left[\begin{array}{ll}
0.9320 & 0.6628 \\
0.6628 & 1.5444
\end{array}\right], \\
G_{\text {amt }}=\left[\begin{array}{ll}
0.9317 & 0.6608 \\
0.6608 & 1.5419
\end{array}\right] .
\end{gathered}
$$

For our geometric mean $G$ (by (2.3))

$$
G=\left[\begin{array}{ll}
0.9319 & 0.6618 \\
0.6618 & 1.5431
\end{array}\right]\left(=A_{1}^{(r)}=A_{2}^{(r)}=A_{3}^{(r)} \text { for } r \geq 3\right) .
$$

\section{Weighted geometric means}

We show two facts with respect to weighted geometric means. First we give

Proposition 3.1. For any operators $A, B$ and for any positive integers $\ell, m \geq 1$

$$
G_{\ell+m}=G_{\ell+m}(\overbrace{A, \cdots, A}^{\ell}, \overbrace{B, \cdots, B}^{m})=A \sharp \frac{m}{\ell+m} B .
$$


Proof. First for $m=1$, we obtain, by induction with respect to $\ell$,

$$
G_{\ell+1}(\overbrace{A, \cdots, A}^{\ell}, B)=A \sharp \frac{1}{\ell+1} B .
$$

In fact, for $\ell=1,(3.2)$ is obvious. Assuming (3.2) (for $\ell$ ), we want to prove

$$
G_{\ell+2}(\overbrace{A, \cdots, A}^{\ell+1}, B)=A \sharp \frac{1}{\ell+2} B .
$$

Put $A_{i}^{(1)}=A$ for $i=1, \cdots, \ell+1$ and $A_{\ell+2}^{(1)}=B$, then

$$
\begin{aligned}
A_{1}^{(2)} & =A \sharp_{\frac{\ell+1}{\ell+2}} G_{\ell+1}\left(A_{2}^{(1)}, \cdots, A_{\ell+1}^{(1)}, A_{\ell+2}^{(1)}\right) \\
& =A \sharp_{\ell+1}^{\ell+2} G_{\ell+1}(\overbrace{A, \cdots, A}^{\ell} B)=A \sharp_{\ell+1}^{\ell+2}\left(A \sharp_{\frac{1}{\ell+1}} B\right)=A \sharp_{\frac{1}{\ell+2}} B .
\end{aligned}
$$

Similarly, we have $A_{2}^{(2)}=\cdots=A_{\ell+1}^{(2)}=A_{\frac{1}{\ell+2}} B$. For $A_{\ell+2}^{(2)}$, we have $A_{\ell+2}^{(2)}=A_{\ell+2}^{(1)} \sharp_{\ell+1}^{\ell+2} G_{\ell+1}\left(A_{2}^{(1)}, \cdots, A_{\ell+1}^{(1)}\right)=B \sharp_{\frac{\ell+1}{\ell+2}} G(A, \cdots, A)=B \sharp_{\frac{\ell+1}{\ell+2}} A=A \sharp_{\frac{1}{\ell+2}} B$.

Hence for $r \geq 3$, we have $A_{i}^{(r)}=A \sharp \frac{1}{\ell+2} B$ for $i=1, \cdots, \ell+2$, so that $G_{\ell+2}=A \sharp \frac{1}{\ell+2} B$ as the limit of $A_{i}^{(r)}(r \rightarrow \infty)$, which is desired.

Now we have obtained (3.1) for $m=1$ and all $\ell \geq 1$. Assuming (3.1) for a fixed $m \geq 1$ and all $\ell \geq 1$, we then have to show, for all $\ell \geq 1$,

$$
G_{\ell+m+1}(\overbrace{A, \cdots, A}^{\ell}, \overbrace{B, \cdots, B}^{m+1})=A \sharp \frac{m+1}{\ell+m+1} B .
$$

For $\ell=1$, we obtain

$$
G_{m+2}(A, \overbrace{B, \cdots, B}^{m+1})=G_{m+2}(\overbrace{B, \cdots, B}^{m+1}, A)=B \sharp \frac{1}{m+2} A=A \sharp \frac{m+1}{m+2} B .
$$

Now assuming (3.3) (for $\ell$ ), we want to prove

$$
G_{\ell+m+2}(\overbrace{A, \cdots, A}^{\ell+1}, \overbrace{B, \cdots, B}^{m+1})=A \sharp \frac{m+1}{\ell+m+2} B .
$$

Put $A_{i}^{(1)}=A$ for $i=1, \cdots, \ell+1$ and $A_{i}^{(1)}=B$ for $i=\ell+2, \cdots, \ell+m+2$. Then for $i=1, \cdots, \ell+1$

$$
A_{i}^{(2)}=A \sharp_{\frac{\ell+m+1}{\ell+m+2}} G_{\ell+m+1}(\overbrace{A, \cdots, A}^{\ell}, \overbrace{B, \cdots, B}^{m+1})=A \sharp \frac{\ell+m+1}{\ell+m+2}\left(A \sharp \frac{m+1}{\ell+m+1} B\right)=A \sharp \frac{m+1}{\ell+m+2} B .
$$


For $i=\ell+2, \cdots, \ell+m+2$

$$
\begin{aligned}
& A_{i}^{(2)}=B \sharp \frac{\ell+m+1}{\ell+m+2} G_{\ell+m+1}(\overbrace{A, \cdots, A}^{\ell+1}, \overbrace{B, \cdots, B}^{m}) \\
& =B \sharp_{\ell+m+1}^{\ell+m+2}\left(A \sharp_{\frac{m}{\ell+m+1}} B\right)=B \sharp_{\frac{\ell+m+1}{\ell+m+2}}\left(B \sharp_{\frac{\ell+1}{\ell+m+1}} A\right)=B \sharp_{\frac{\ell+1}{\ell+m+2}} A=A \sharp_{\frac{m+1}{\ell+m+2}} B .
\end{aligned}
$$

Hence for $r \geq 2$ we have $A_{i}^{(r)}=A \sharp_{\frac{m+1}{\ell+m+2}} B$ for all $i=1, \cdots, \ell+m+2$, which is desired.

Next we define a weighted geometric mean of three positive operators. Let $\alpha, \beta, \gamma$ be real numbers such that

$$
\alpha, \beta, \gamma \geq 0 \text { and } \alpha+\beta+\gamma=1 .
$$

Then for operators $A, B, C$ and real numbers $\alpha, \beta, \gamma$ satisfying (3.4), we define the sequences $\left\{A_{n}\right\},\left\{B_{n}\right\}$ and $\left\{C_{n}\right\}$ as follows:

$$
\begin{gathered}
A_{1}=A, \quad B_{1}=B, \quad C_{1}=C \text { and for } n \geq 1 \\
\qquad \begin{array}{l}
A_{n+1}=A_{n} \sharp \alpha_{1}\left(B_{n} \sharp \alpha_{2} C_{n}\right), \\
B_{n+1}=B_{n} \sharp \beta_{1}\left(C_{n} \sharp \beta_{2} A_{n}\right), \\
C_{n+1}=C_{n} \sharp \gamma_{1}\left(A_{n} \sharp \gamma_{2} B_{n}\right) .
\end{array}
\end{gathered}
$$

Here the above constants are:

$$
\alpha_{1}=1-\alpha, \alpha_{2}=1-\frac{\beta}{\alpha_{1}}, \beta_{1}=1-\beta, \beta_{2}=1-\frac{\gamma}{\beta_{1}}, \gamma_{1}=1-\gamma, \gamma_{2}=1-\frac{\alpha}{\gamma_{1}}
$$

with a convention $\frac{y}{1-x}=0$ for $x=1$. These are the solutions for the equations

$$
\left\{\begin{array}{l}
\alpha=1-\alpha_{1}=\beta_{1} \beta_{2}=\gamma_{1}\left(1-\gamma_{2}\right), \\
\beta=\alpha_{1}\left(1-\alpha_{2}\right)=1-\beta_{1}=\gamma_{1} \gamma_{2}, \\
\gamma=\alpha_{1} \alpha_{2}=\beta_{1}\left(1-\beta_{2}\right)=1-\gamma_{1} .
\end{array}\right.
$$

which are obtained by observing the exponents for commuting operators:

$$
\begin{aligned}
& A_{n+1}=A_{n}^{1-\alpha_{1}} B_{n}^{\alpha_{1}\left(1-\alpha_{2}\right)} C_{n}^{\alpha_{1} \alpha_{2}}, \\
& B_{n+1}=B_{n}^{1-\beta_{1}} C_{n}^{\beta_{1}\left(1-\beta_{2}\right)} A_{n}^{\beta_{1} \beta_{2}}, \\
& C_{n+1}=C_{n}^{1-\gamma_{1}} A_{n}^{\gamma_{1}\left(1-\gamma_{2}\right)} B_{n}^{\gamma_{1} \gamma_{2}} .
\end{aligned}
$$

Now we obtain a weighted geometric mean as the common limit of $\left\{A_{n}\right\},\left\{B_{n}\right\}$ and $\left\{C_{n}\right\}$ : 
Theorem 3.2. Let $\left\{A_{n}\right\},\left\{B_{n}\right\}$ and $\left\{C_{n}\right\}$ be the sequences given by (3.5) for operators $A, B, C$ and real numbers $\alpha_{i}, \beta_{i}, \gamma_{i}, \alpha, \beta, \gamma$ satisfying (3.6). Then the sequences converge and have a common limit, which we denote by $G(A, B, C ; \alpha, \beta, \gamma)$. Moreover, the limit is permutation invariant, that is,

$$
G(A, B, C ; \alpha, \beta, \gamma)=G(\pi(A, B, C) ; \pi(\alpha, \beta, \gamma))
$$

for any permutations $\pi(A, B, C), \pi(\alpha, \beta, \gamma)$ of $(A, B, C),(\alpha, \beta, \gamma)$, respectively.

Lemma 3.3. Let $\left\{A_{n}\right\},\left\{B_{n}\right\}$ and $\left\{C_{n}\right\}$ be the sequences given by (3.5) and $\alpha, \beta, \gamma$, $\alpha_{i}, \beta_{i}, \gamma_{i}(i=1,2)$ be real numbers satisfying (3.6). Then

$$
\left\{\begin{array}{l}
d\left(A_{n+1}, B_{n+1}\right) \leq 2 \min \{\alpha, \beta\} d\left(A_{n}, B_{n}\right) \leq(2 M)^{n} d(A, B), \\
d\left(B_{n+1}, C_{n+1}\right) \leq 2 \min \{\beta, \gamma\} d\left(B_{n}, C_{n}\right) \leq(2 M)^{n} d(B, C), \\
d\left(C_{n+1}, A_{n+1}\right) \leq 2 \min \{\gamma, \alpha\} d\left(C_{n}, A_{n}\right) \leq(2 M)^{n} d(C, A),
\end{array}\right.
$$

where

$$
M=\max \{\min \{\alpha, \beta\}, \min \{\beta, \gamma\}, \min \{\gamma, \alpha\}\},
$$

or, is the second number of $\alpha, \beta, \gamma$ in size.

Proof. By the definition (3.5), we have

$$
\begin{aligned}
& d\left(A_{n+1}, B_{n+1}\right)=d\left(A_{n} \sharp_{\alpha_{1}}\left(B_{n} \sharp_{\alpha_{2}} C_{n}\right), B_{n} \sharp_{\beta_{1}}\left(C_{n} \sharp_{\beta_{2}} A_{n}\right)\right) \\
\leq & d\left(A_{n} \sharp_{\alpha_{1}}\left(B_{n} \sharp_{\alpha_{2}} C_{n}\right), A_{n} \sharp_{\beta_{1}}\left(C_{n} \sharp_{\beta_{2}} A_{n}\right)\right)+d\left(A_{n} \sharp_{\beta_{1}}\left(C_{n} \sharp_{\beta_{2}} A_{n}\right), B_{n} \sharp_{\beta_{1}}\left(C_{n} \sharp_{\beta_{2}} A_{n}\right)\right) \\
& (:=I+I I) .
\end{aligned}
$$

Note that from WG3 and WG11,

$$
A_{n} \sharp_{\beta_{1}}\left(C_{n} \sharp_{\beta_{2}} A_{n}\right)=A_{n} \sharp_{\beta_{1}}\left(A_{n} \sharp_{1-\beta_{2}} C_{n}\right)=A_{n} \sharp_{\beta_{1}\left(1-\beta_{2}\right)} C_{n}=A_{n} \sharp_{\alpha_{1}}\left(A_{n} \sharp_{\alpha_{2}} C_{n}\right) .
$$

Hence we have

$$
\begin{aligned}
& I:=d\left(A_{n} \sharp_{\alpha_{1}}\left(B_{n} \sharp_{\alpha_{2}} C_{n}\right), A_{n} \sharp_{\beta_{1}}\left(C_{n} \sharp_{\beta_{2}} A_{n}\right)\right)=d\left(A_{n} \sharp_{\alpha_{1}}\left(B_{n} \sharp_{\alpha_{2}} C_{n}\right), A_{n} \sharp \alpha_{1}\left(A_{n} \sharp \alpha_{2} C_{n}\right)\right) \\
& \leq\left(1-\alpha_{1}\right) d\left(A_{n}, A_{n}\right)+\alpha_{1} d\left(B_{n} \sharp \alpha_{2} C_{n}, A_{n} \sharp_{\alpha_{2}} C_{n}\right) \leq \alpha_{1}\left(1-\alpha_{2}\right) d\left(B_{n}, A_{n}\right)=\beta d\left(A_{n}, B_{n}\right) . \\
& I I:=d\left(A_{n} \sharp_{\beta_{1}}\left(C_{n} \sharp_{\beta_{2}} A_{n}\right), B_{n} \sharp_{\beta_{1}}\left(C_{n} \sharp_{\beta_{2}} A_{n}\right)\right) \\
& \leq\left(1-\beta_{1}\right) d\left(A_{n}, B_{n}\right)+\beta_{1} d\left(C_{n} \sharp_{\beta_{2}} A_{n}, C_{n} \sharp_{\beta_{2}} A_{n}\right)=\beta d\left(A_{n}, B_{n}\right) .
\end{aligned}
$$

Hence

$$
d\left(A_{n+1}, B_{n+1}\right) \leq I+I I \leq 2 \beta d\left(A_{n}, B_{n}\right) .
$$

Again

$$
\begin{aligned}
& d\left(A_{n+1}, B_{n+1}\right)=d\left(A_{n} \sharp \alpha_{1}\left(B_{n} \sharp_{\alpha_{2}} C_{n}\right), B_{n} \sharp_{\beta_{1}}\left(C_{n} \sharp \beta_{2} A_{n}\right)\right) \\
& \leq d\left(A_{n} \sharp \alpha_{1}\left(B_{n} \sharp \alpha_{2} C_{n}\right), B_{n} \sharp \alpha_{1}\left(B_{n} \sharp_{\alpha_{2}} C_{n}\right)\right)+d\left(B_{n} \sharp \alpha_{1}\left(B_{n} \sharp \alpha_{2} C_{n}\right), B_{n} \sharp \beta_{1}\left(C_{n} \sharp \beta_{2} A_{n}\right)\right) \\
& \quad(:=I I I+I V) .
\end{aligned}
$$


Note that

$$
B_{n} \sharp_{\alpha_{1}}\left(B_{n} \sharp_{\alpha_{2}} C_{n}\right)=B_{n} \sharp_{\alpha_{1} \alpha_{2}} C_{n}=B_{n} \sharp_{\beta_{1}}\left(C_{n} \sharp_{\beta_{2}} B_{n}\right) .
$$

So that

$$
\begin{aligned}
& I I I:=d\left(A_{n} \sharp_{\alpha_{1}}\left(B_{n} \sharp_{\alpha_{2}} C_{n}\right), B_{n} \sharp_{\alpha_{1}}\left(B_{n} \sharp_{\alpha_{2}} C_{n}\right)\right) \\
& \leq\left(1-\alpha_{1}\right) d\left(A_{n}, B_{n}\right)+\alpha_{1} d\left(B_{n} \sharp_{\alpha_{2}} C_{n}, B_{n} \sharp_{\alpha_{2}} C_{n}\right)=\alpha d\left(A_{n}, B_{n}\right) . \\
& I V:=d\left(B_{n} \sharp \alpha_{1}\left(B_{n} \sharp \alpha_{2} C_{n}\right), B_{n} \sharp_{\beta_{1}}\left(C_{n} \sharp_{\beta_{2}} A_{n}\right)\right) \\
& \quad=d\left(B_{n} \sharp_{\beta_{1}}\left(C_{n} \sharp_{\beta_{2}} B_{n}\right), B_{n} \sharp_{\beta_{1}}\left(C_{n} \sharp_{\beta_{2}} A_{n}\right)\right) \\
& \leq\left(1-\beta_{1}\right) d\left(B_{n}, B_{n}\right)+\beta_{1}\left(\left(1-\beta_{2}\right) d\left(C_{n}, C_{n}\right)+\beta_{2} d\left(B_{n}, A_{n}\right)\right)=\alpha d\left(A_{n}, B_{n}\right) .
\end{aligned}
$$

From the above inequalities we have

$$
d\left(A_{n+1}, B_{n+1}\right) \leq I I I+I V \leq 2 \alpha d\left(A_{n}, B_{n}\right) .
$$

Now from (3.9) and (3.10), we have $d\left(A_{n+1}, B_{n+1}\right) \leq 2 \min \{\alpha, \beta\} d\left(A_{n}, B_{n}\right)$, which implies the first inequalities of (3.7).

Similarly we can show the second and the third inequalities.

Lemma 3.4. Let $\left\{A_{n}\right\},\left\{B_{n}\right\}$ and $\left\{C_{n}\right\}$ be sequences given by (3.5) and let $\alpha, \beta, \gamma$, $\alpha_{i}, \beta_{i}, \gamma_{i}(i=1,2)$ be real numbers satisfying (3.6). Then

$$
\left\{\begin{array}{l}
d\left(A_{n+1}, A_{n}\right) \leq \beta d\left(A_{n}, B_{n}\right)+\gamma d\left(C_{n}, A_{n}\right) \leq(2 M)^{n-1}\{\beta d(A, B)+\gamma d(C, A)\}, \\
d\left(B_{n+1}, B_{n}\right) \leq \gamma d\left(B_{n}, C_{n}\right)+\alpha d\left(A_{n}, B_{n}\right) \leq(2 M)^{n-1}\{\gamma d(B, C)+\alpha d(A, B)\}, \\
d\left(C_{n+1}, C_{n}\right) \leq \alpha d\left(C_{n}, A_{n}\right)+\beta d\left(B_{n}, C_{n}\right) \leq(2 M)^{n-1}\{\alpha d(C, A)+\beta d(B, C)\},
\end{array}\right.
$$

where $M$ is defined in (3.8).

Proof. Using WG0, we have

$$
\begin{aligned}
& d\left(A_{n+1}, A_{n}\right)=d\left(A_{n} \sharp \alpha_{1}\left(B_{n} \sharp \alpha_{2} C_{n}\right), A_{n} \sharp \alpha_{1}\left(A_{n} \sharp \alpha_{2} A_{n}\right)\right) \\
& \quad \leq\left(1-\alpha_{1}\right) d\left(A_{n}, A_{n}\right)+\alpha_{1} d\left(B_{n} \sharp \alpha_{2} C_{n}, A_{n} \sharp \alpha_{2} A_{n}\right) \\
& \quad \leq \alpha_{1}\left(1-\alpha_{2}\right) d\left(A_{n}, B_{n}\right)+\alpha_{1} \alpha_{2} d\left(C_{n}, A_{n}\right) \\
& \quad=\beta d\left(A_{n}, B_{n}\right)+\gamma d\left(C_{n}, A_{n}\right) \leq(2 M)^{n-1}\{\beta d(A, B)+\gamma d(C, A)\} .
\end{aligned}
$$

In the same manner, we can obtain other inequalities in (3.11).

Proof of Theorem 3.2. We may only consider the case that all of $\alpha, \beta$ and $\gamma$ are nonzero or smaller than 1 , so that we can assume $M<1 / 2$. Then from Lemmas 3.3 and 3.4 we can show that the sequences $\left\{A_{n}\right\},\left\{B_{n}\right\}$ and $\left\{C_{n}\right\}$ converge and have a common limit by using the similar argument as in the proof of Theorem 2.2. For the property of permutation invariance of the limit $G(A, B, C ; \alpha, \beta, \gamma)$, we can also show the fact almost similarly as in the proof of Theorem 2.2.

The following result implies that $G(A, B, C ; \alpha, \beta, \gamma)$ is really an extension of the weighted mean of two operators: 
Proposition 3.5. Let $A$ and $B$ be positive operators, and let $\alpha, \beta, \gamma$ be real numbers satisfying (3.4). Then

$$
G(A, A, B ; \alpha, \beta, \gamma)=A \sharp_{\gamma} B .
$$

Proof. In (3.5), replace $A_{1}=B_{1}=A$ and $C_{1}=B$, then

$$
A_{2}=A \sharp_{\alpha_{1}}\left(A \sharp_{\alpha_{2}} B\right)=A \sharp_{\alpha_{1} \alpha_{2}} B=A \sharp_{\gamma} B .
$$

Similarly, we can obtain $B_{2}=C_{2}=A \sharp_{\gamma} B$, so that

$$
A_{n}=B_{n}=C_{n}=A \sharp_{\gamma} B \text { for } n \geq 2 .
$$

This implies the desired identity (3.12).

Kosaki [14] presented the following definition of a weighted geometric mean $\tilde{G}_{K}=\tilde{G}_{K}^{+} \sharp \tilde{G}_{K}^{-}$. Here $\tilde{G}_{K}^{+}=\tilde{G}_{K}^{+}\left(A_{1}, \cdots, A_{k} ; \alpha_{1}, \cdots, \alpha_{k}\right)$ is defined as the extended form of $(2.6)$ :

$$
\tilde{G}_{K}^{+}=\frac{1}{\Pi_{j=1}^{k} \Gamma\left(\alpha_{j}\right)} \int_{\Lambda_{k}}\left\{\sum_{j=1}^{k} \lambda_{j} A_{j}^{-1}\right\}^{-1}\left\{\sum_{j=1}^{k} \lambda_{j}\right\}\left\{\prod_{j=1}^{k} \lambda_{j}^{1 / \alpha_{j}-1}\right\} d \lambda_{1} \cdots d \lambda_{k},
$$

and $\tilde{G}_{K}^{-}=\left(\tilde{G}_{K}^{+}\right)^{*}$, the dual of $\tilde{G}_{K}^{+}$.

For numerical computation of $\tilde{G}_{K}$ and $\tilde{G}$, the weighted geometric mean by our definition, take three matrices

$$
A=\left[\begin{array}{ll}
2 & 1 \\
1 & 1
\end{array}\right], B=\left[\begin{array}{ll}
1 & 1 \\
1 & 2
\end{array}\right] \text { and } C=\left[\begin{array}{cc}
3 & \sqrt{2} \\
\sqrt{2} & 1
\end{array}\right] .
$$

Then for $\alpha=1 / 2, \beta=1 / 3, \gamma=1 / 6$ we have, by the Simpson's formula for the integral on the interval $[0,1]$ divided into $2 \times 10^{4}$ segments,

$$
\tilde{G}_{K}=\left[\begin{array}{ll}
1.6119 & 0.9374 \\
0.9374 & 1.1655
\end{array}\right]\left(=\tilde{G}_{K, 1}\right) .
$$

From another computation by using Gauss-Legendre quadrature with 150 nodes, we have

$$
\tilde{G}_{K}=\left[\begin{array}{ll}
1.6118 & 0.9375 \\
0.9375 & 1.1648
\end{array}\right]\left(=\tilde{G}_{K, 2}\right) .
$$

Hence it seems that the true $\tilde{G}_{k}$ is a matrix with an error at most $10^{-3}$ for each component of $\tilde{G}_{k, 1}$ or $\tilde{G}_{k, 2}$. For $\tilde{G}$ by our definition we have

$$
\tilde{G}=\left[\begin{array}{ll}
1.6185 & 0.9375 \\
0.9375 & 1.1608
\end{array}\right]\left(=A_{1}^{(r)}=A_{2}^{(r)}=A_{3}^{(r)} \text { for } r \geq 3\right) .
$$




\section{Reverse inequality}

Recently Kantorovich type reverse inequalities of the arithmetic-geometric, the arithmetic-harmonic, or the arithmetic-geometric-harmonic ones for two or more than two operators were presented in [7], [9], [19]. The following fact was shown in [9]:

Lemma 4.1 ([9, Theorem 9]). Let $A_{1}, A_{2}, \cdots, A_{n}$ be operators such that $0<m I \leq$ $A_{i} \leq M I$ for $i=1,2, \cdots, n$ for some scalars $m$ and $M$ with $0<m<M$. (The letter I stands the identity operator.) Then

$$
\frac{A_{1}+\cdots+A_{n}}{n} \leq \frac{(M+m)^{2}}{4 M m}\left(\frac{A_{1}^{-1}+\cdots+A_{n}^{-1}}{n}\right)^{-1} .
$$

From the above lemma and the property P10 of our geometric mean, we immediately obtain the following result:

Proposition 4.2. Let $A_{1}, A_{2}, \cdots, A_{n}$ be operators such that $0<m I \leq A_{i} \leq M I$ for $i=1,2, \cdots, n$ for some scalars $m$ and $M$ with $0<m<M$. Then

$$
\frac{A_{1}+A_{2}+\cdots+A_{n}}{n} \leq \frac{(M+m)^{2}}{4 M m} G\left(A_{1}, A_{2}, \cdots, A_{n}\right) .
$$

For the weighted version of the arithmetic-geometric-harmonic mean inequality we can show for operators $A, B, C$ and real numbers $\alpha, \beta, \gamma$ with the assumption (3.4),

$$
\alpha A+\beta B+\gamma C \geq G(A, B, C ; \alpha, \beta, \gamma) \geq\left(\alpha A^{-1}+\beta B^{-1}+\gamma C^{-1}\right)^{-1} .
$$

As a reverse version of the arithmetic-geometric mean inequality, we can obtain

Proposition 4.3. Let $m I \leq A, B, C \leq M I$ for some scalars $m$ and $M$ with $0<m<M$. Then with the assumption (3.4) for real numbers $\alpha, \beta, \gamma$,

$$
\alpha A+\beta B+\gamma C \leq \frac{(M+m)^{2}}{4 M m} G(A, B, C ; \alpha, \beta, \gamma) .
$$

Acknowledgment. The author would like to thank Professor S. Izumino for valuable suggestions.

\section{References}

[1] W. N. Anderson, Jr., T. D. Morley and G.E. Trapp, Symmetric function means of positive operators, Linear Algebra Appl., 60(1984), 129-143. 
[2] T. Ando, Topics on operator inequalities, Hokkaido Univ. Lecture Note, 1978.

[3] T. Ando, C.-K. Li and R. Mathias, Geometric means, Linear Algebra Appl., 385(2004), 305-334.

[4] E. Andruchow, G. Corach and D. Stojanoff, Geometrical significance of the LöwnerHeinz inequality, Proc. Amer. Math. Soc., 128(1999), 1031-1037.

[5] G. Corach, H. Porta and L. Recht, Convexity of the geodestic distance on spaces of positive operators, Illinois J. Math., 38(1994), 87-94.

[6] B. Q. Feng and A. Tonge, Geometric means and Hadamard products, Math. Inequalities Appl., 8(2005), 559-564.

[7] J. I. Fujii, M. Fujii, M. Nakamura, J. Pečarić and Y. Seo, A reverse inequality for the weighted geometric mean due to Lawson-Lim, Linear Algebra Appl., 427(2007), $272-284$.

[8] J. I. Fujii and T. Furuta, An operator version of the Wilf-Diaz-Metcalf inequality, Nihonkai Math. J., 9(1998), 47-52.

[9] J. I. Fujii, M. Nakamura, J. Pečarić and Y. Seo, Bounds for the ratio and difference between parallel sum and series via Mond-Pečarić method, Math. Inequalities and Appl., 9(2006), 749-759.

[10] M. Fujii, S. Izumino, R. Nakamoto and Y. Seo, Operator inequalities related to Cauchy-schwarz and Hölder-McCarthy inequalities, Nihonkai Math. J., 8(1997), 117122.

[11] M. Fujii, J. F. Jiang and E. Kamei, A geometrical structure in the Furuta inequality II, Nihonkai Math. J., 8(1997), 37-46.

[12] M. Fujii and E. Kamei, Mean theoretic approach to the grand Furuta inequality, Proc. Amer. Math. Soc., 124(1996), 2751-2756.

[13] T. Furuta, J. Mićić, J. Pečarić and Y. Seo, Mond-Pečarić Method in Operator Inequalities, Monographs in Inequalities I, Element, Zagreb, 2005.

[14] H. Kosaki, Geometric mean of several positive operators, 1984.

[15] F. Kubo, and T. Ando, Means of positive linear operators, Math. Ann., 246(1980), 205-224.

[16] J. Lawson and Y. Lim, A general framework for extending means to higher orders, preprint. http : //arxiv.org/PS_cache/math/pdf/0612/0612293v1.pdf.

[17] N. Nakamura, Geometric operator mean induced from the Riccati equation, Sci. Math. Japon., 66(2007), 83-87.

[18] R. D. Nussbaum, Hilbert's projective metric and iterated nonlinear maps, Mem. Amer. Math. Soc., 75(391)(1988).

[19] T. YAMAZAKI, An extension of Kantorovich inequality to $n$-operators via the geometric mean by Ando-Li-Mathias, Linear Algebra Appl., 416(2006), 688-695. 